\title{
Selective Reduction Mechanism of Graphene Oxide Driven by the Photon Mode versus the Thermal Mode
}

\section{Supporting information}

Masaki Hada*, Kiyoshi Miyata, Satoshi Ohmura, Yusuke Arashida, Kohei Ichiyanagi, Ikufumi Katayama, Takayuki Suzuki, Wang Chen, Shota Mizote, Takayoshi Sawa, Takayoshi Yokoya, Toshio Seki, Jiro Matsuo, Tomoharu Tokunaga, Chihiro Itoh, Kenji Tsuruta, Ryo Fukaya, Shunsuke Nozawa, Shin-ichi Adachi, Jun Takeda, Ken Onda*, Shin-ya Koshihara, Yasuhiko Hayashi, and Yuta Nishina*

*Emails: hada.masaki.fm@u.tsukuba.ac.jp (Masaki Hada),

konda@chem.kyushu-univ.jp (Ken Onda),

nisina-y@cc.okayama-u.ac.jp (Yuta Nishina)

\section{Table of Contents}

1. Static characterization of graphene oxide (Figures S1 - S14, and Table S1)

2. DFT and MM2 calculations for graphene oxide (Figures S15 - S18)

3. Transient absorption of graphene oxide (Figures S19 - S21)

4. TD-DFT calculations (Figures S20 - S27, and Note S1)

Supporting References 


\section{Static characterization of graphene oxide}

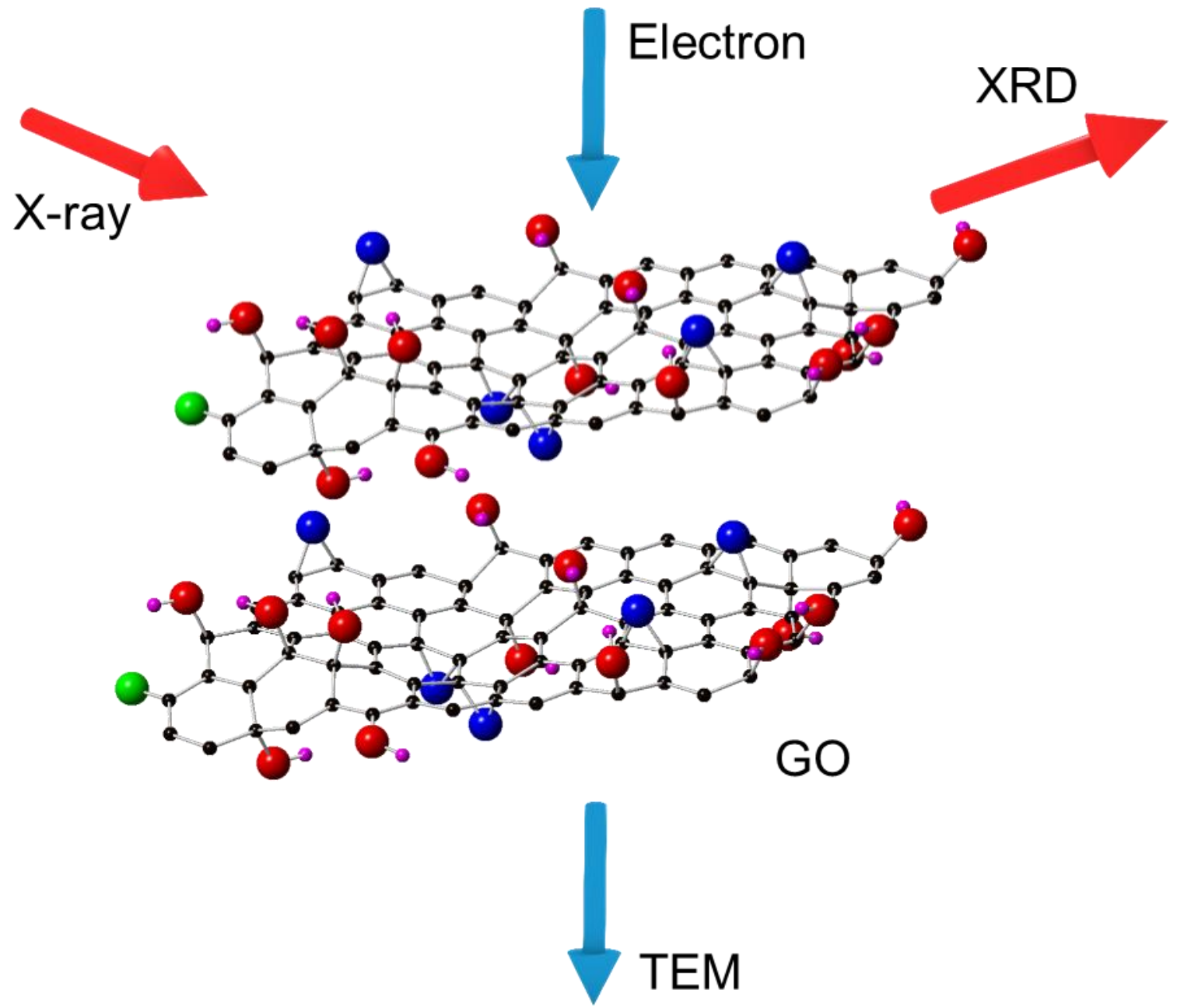

Figure S1. Schematic showing of GO and the geometry of GO used for static measurements (XRD) and electron diffraction using TEM. For the XRD measurements in reflection mode, GO is coated on glass substrates. For the electron diffraction measurements in transmission mode, GO is coated on $\mathrm{SiN}$ membrane substrates. The black, pink, red, blue, green balls represent carbon atoms, hydrogen atoms, and oxygen atoms in hydroxyl groups, oxygen atoms in epoxy groups, and oxygen atoms in carbonyl groups, respectively. The sheet of GO is a stacked single layer, indicating that the GO layers do not have lattice correlations to each other. 


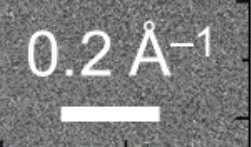

Figure S2. Reflection-mode synchrotron $\mathrm{XRD}^{\mathrm{S} 1, \mathrm{~S} 2}$ image of GO, with the white arrow showing the diffraction spot corresponding to the (0001) plane of GO. From the reflection mode, only the diffraction from the (0001) plane was obtained from GO, suggesting that the planes of GO are parallel to the substrate and are stacked on top of each other. 


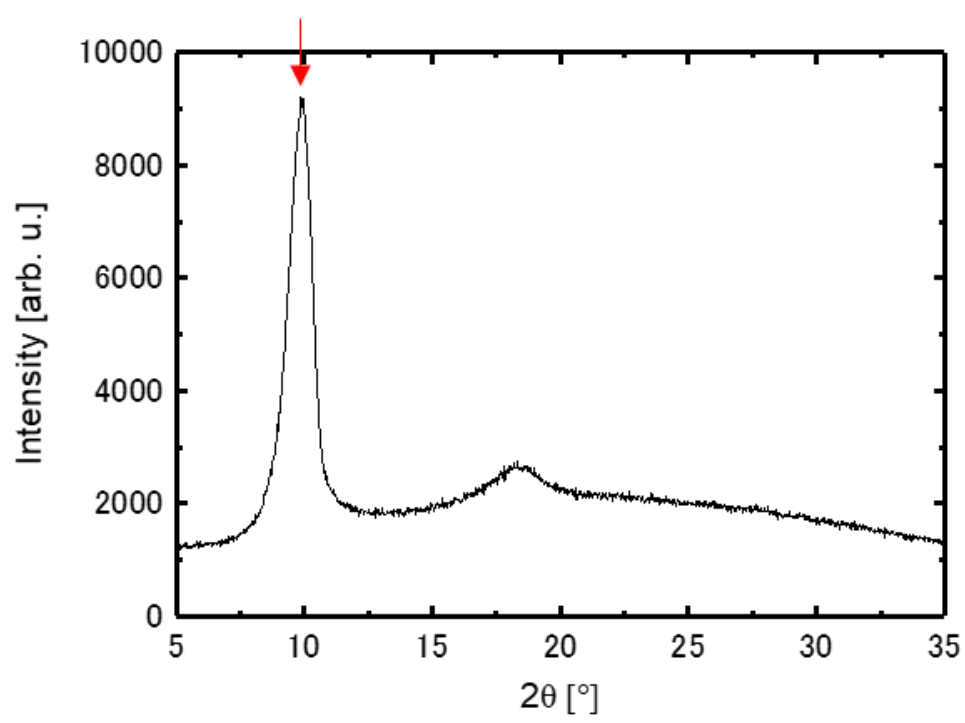

Figure S3. Powder XRD profile of GO. The peak indicated by the red arrow is ascribed to the (0001) plane of GO $\left(2 \theta \sim 10^{\circ}\right)$. According to the XRD profiles of GO, the lattice distance of the (0001) plane is calculated to be $\sim 9 \AA$ using the Bragg's equation $(2 \sin \theta=n \lambda)$. The broad peak at $2 \theta \sim 17^{\circ}$ is attributed to the glass substrate. 


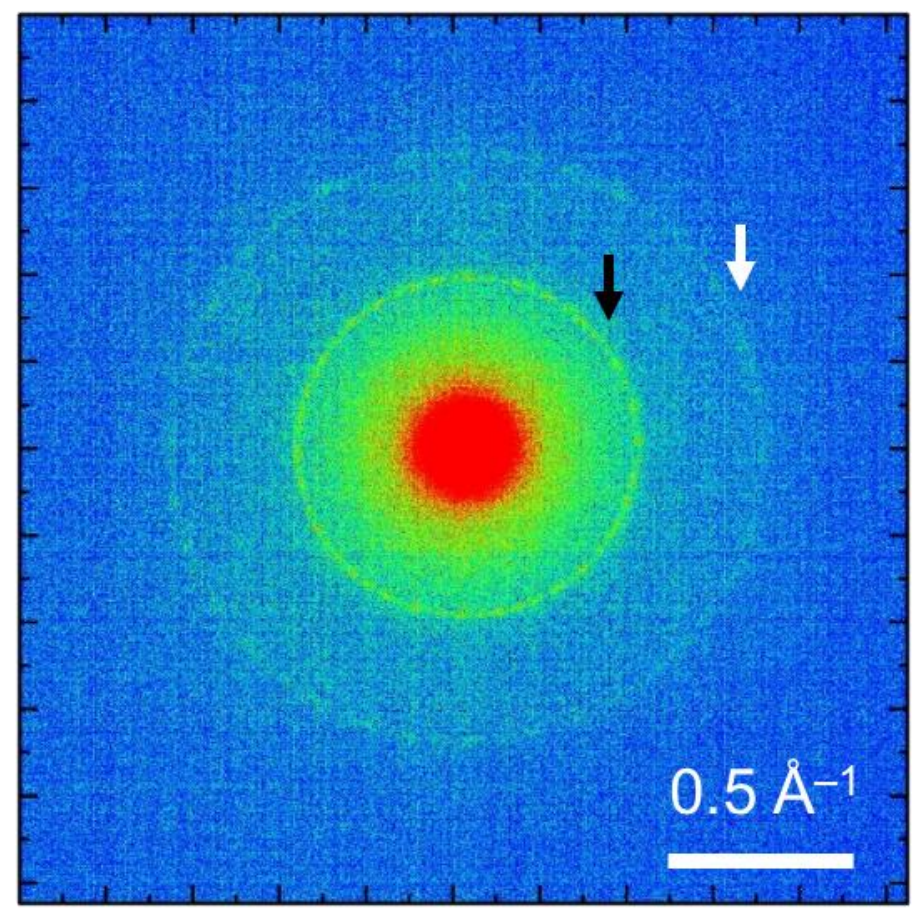

Figure S4. Electron diffraction image acquired using TEM in transmission mode, in which the black and white arrows indicate the diffraction rings corresponding to the

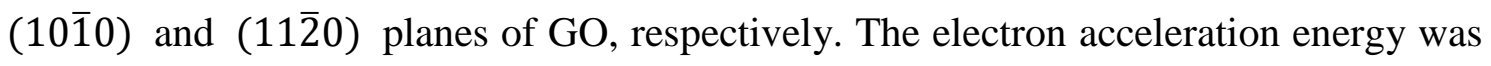
set to $80 \mathrm{keV}$ to suppress electron impact-induced damage to $\mathrm{GO}{ }^{\mathrm{S3}}$ 


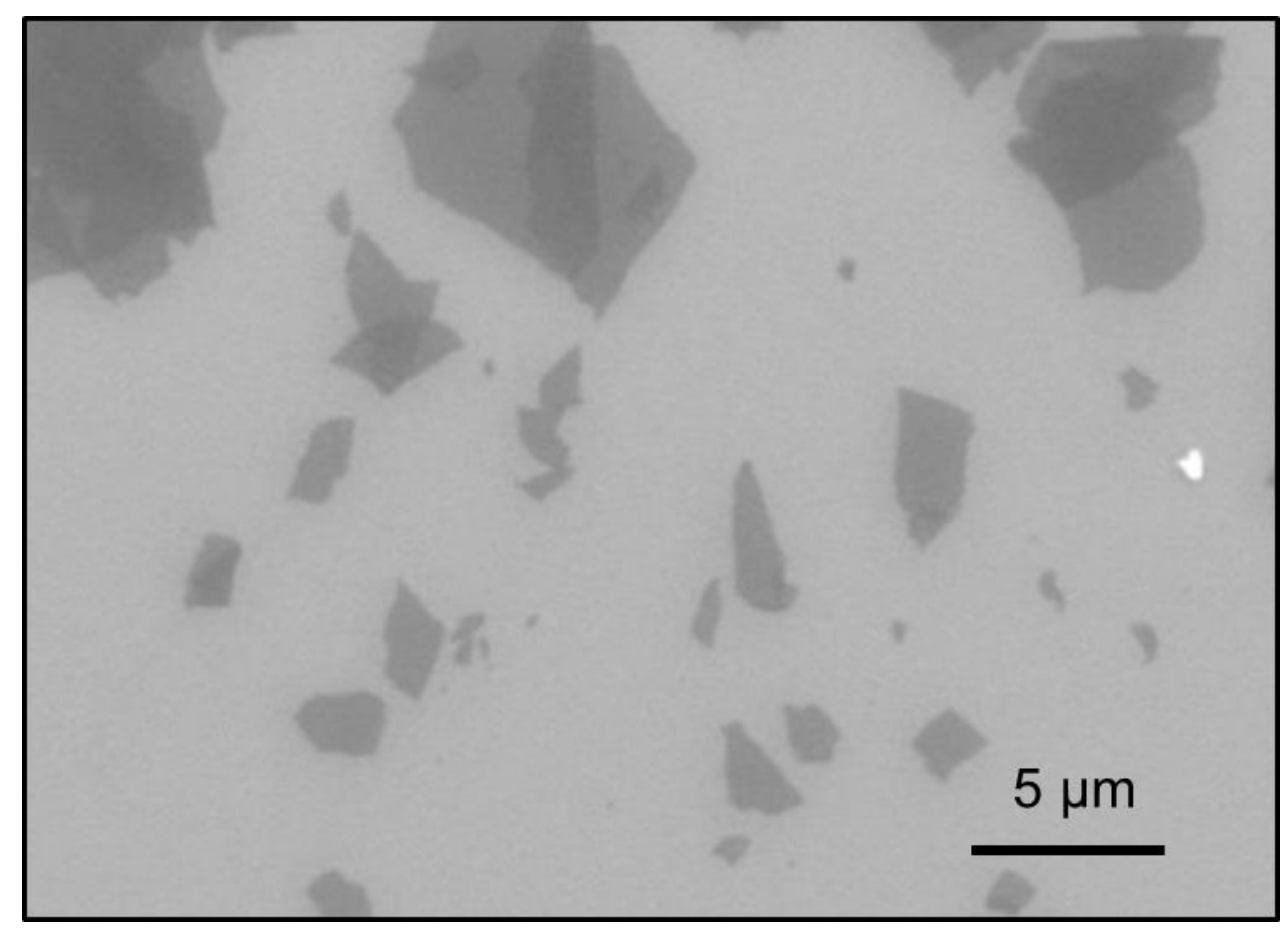

Figure S5. SEM image of GO. The image corresponds to the results obtained from XRD measurements, and thus the planes of GO are parallel to the substrate and stacked on top of each other. 


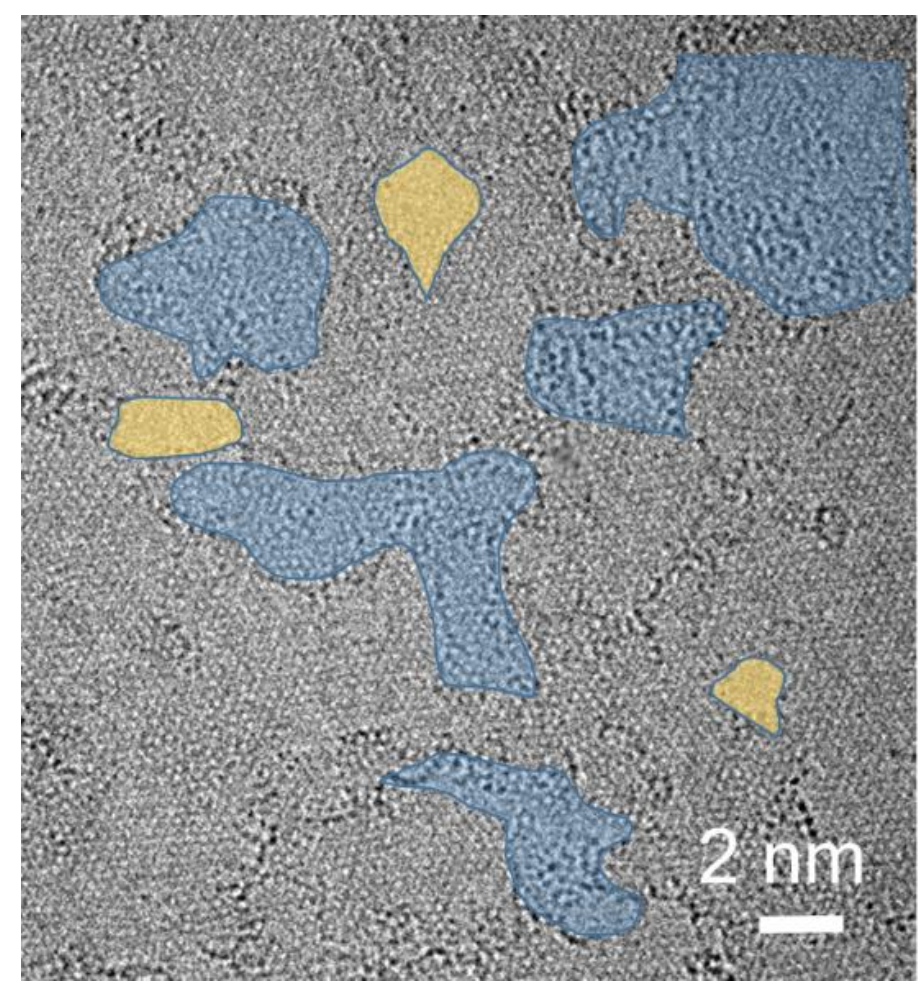

Figure S6. High-resolution TEM image of a single layer of GO. The electron acceleration energy was set to $80 \mathrm{keV}$ to suppress electron impact-induced damage to GO. ${ }^{\mathrm{S} 3}$ The observed images resembled images of rGO previously reported by Gómez-Navarro et $a l .{ }^{\mathrm{S} 3} \mathrm{GO}$ should have three main distinct areas, i.e., a fully oxidized area (blue), an area that retains a graphitic structure (gray), and holes (yellow). 
a
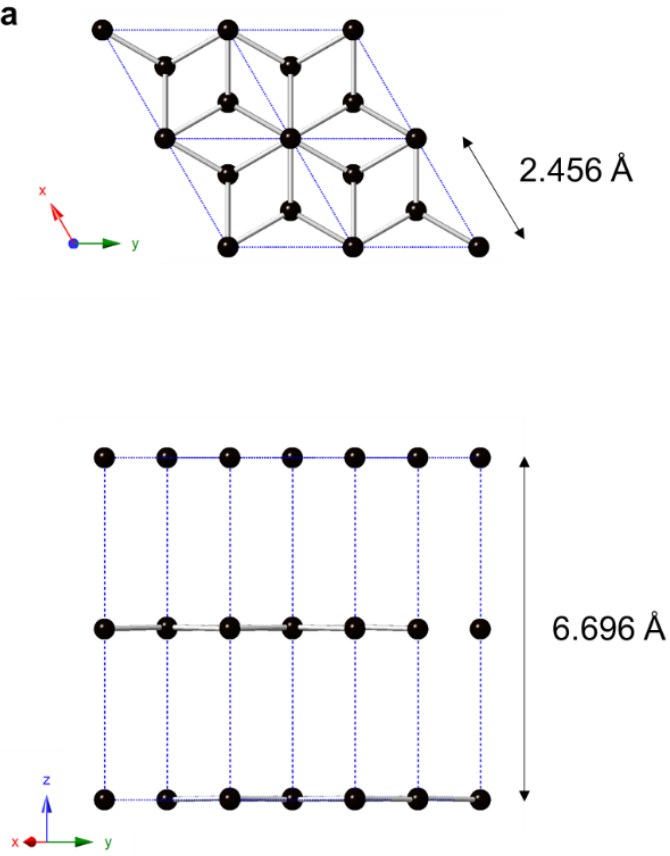

b

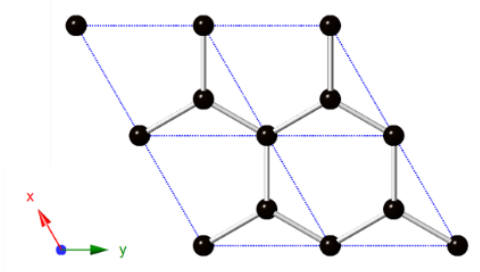

$(0001)$

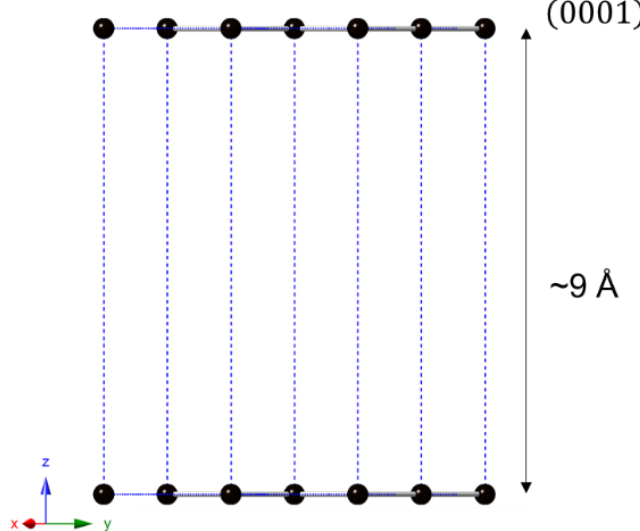

Figure S7. Lattice model of GO. Graphene and GO are two-dimensional materials, and three-dimensional model lattices for graphene and GO have not been reported. We modified the hexagonal lattice of graphite (a) to create the lattice model of GO. The hexagonal lattice of graphite resides in space group of $P 6_{3} m c$, and the lengths of $a$-and $c$-axes are 2.456 and $6.696 \AA$, respectively. ${ }^{\mathrm{S} 4}$ The GO layers do not have lattice correlations with each other, and the distance of the (0001) plane is $\sim 9 \AA$. Thus, the model of the GO structure is presented in (b), where the oxygen functional groups are simplified. 

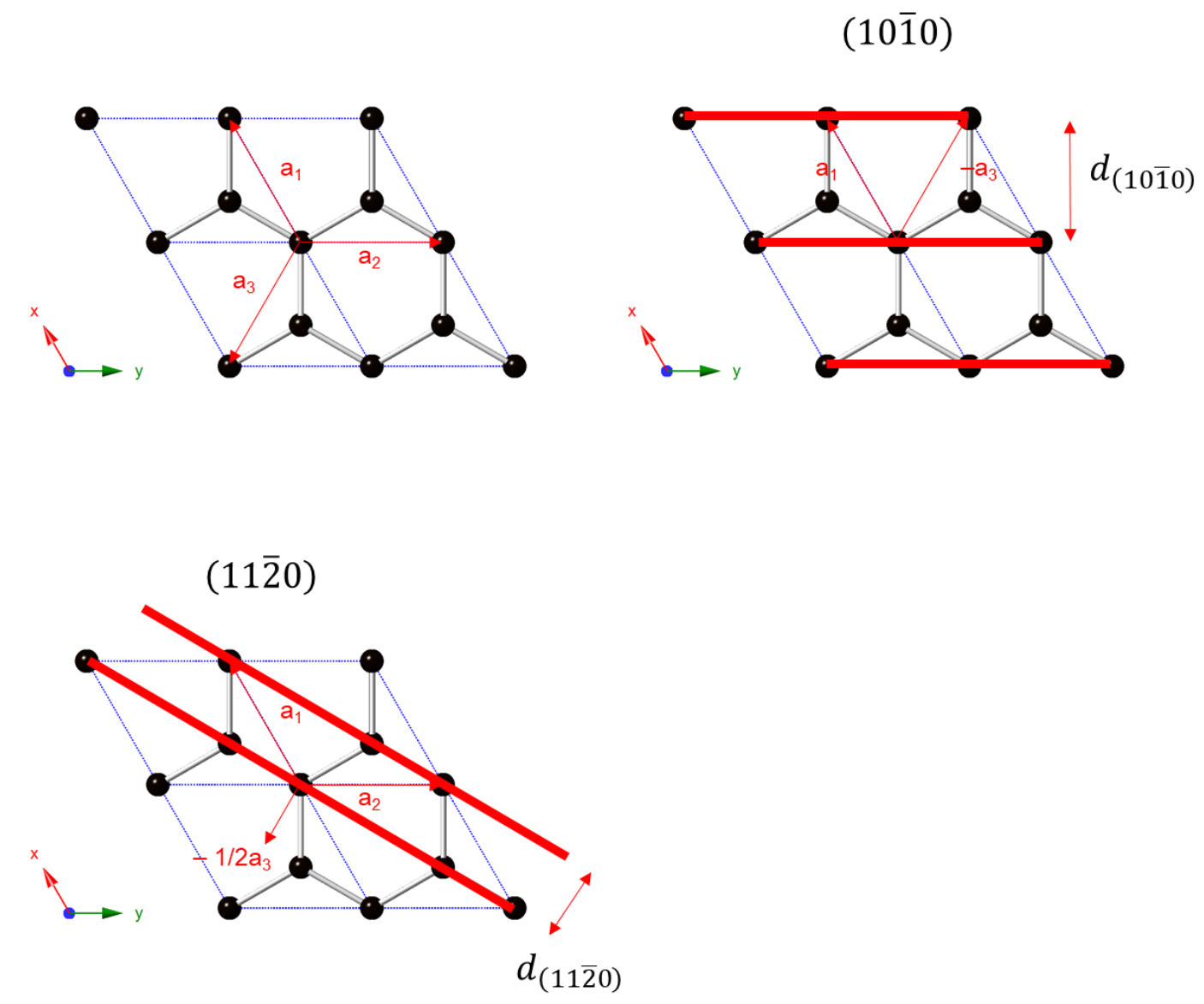

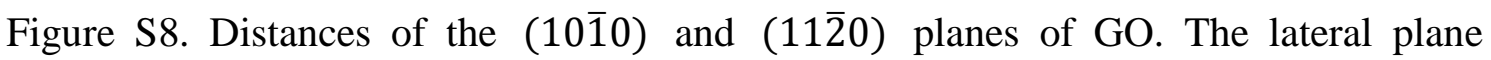
distances were determined from the lattice model of GO shown in Figure S7. According to the geometry, the average $\mathrm{C}-\mathrm{C}$ bond length $\left(d_{\mathrm{C}-\mathrm{C}}\right)$ is calculated from the distances of the $(10 \overline{1} 0)\left(d_{(10 \overline{1} 0)}\right)$ and $(11 \overline{2} 0)\left(d_{(11 \overline{2} 0)}\right)$ planes using the following equation: $d_{\mathrm{C}-\mathrm{C}}=\frac{2}{3} d_{(10 \overline{1} 0)}=\frac{2}{\sqrt{3}} d_{(11 \overline{2} 0)}$. 


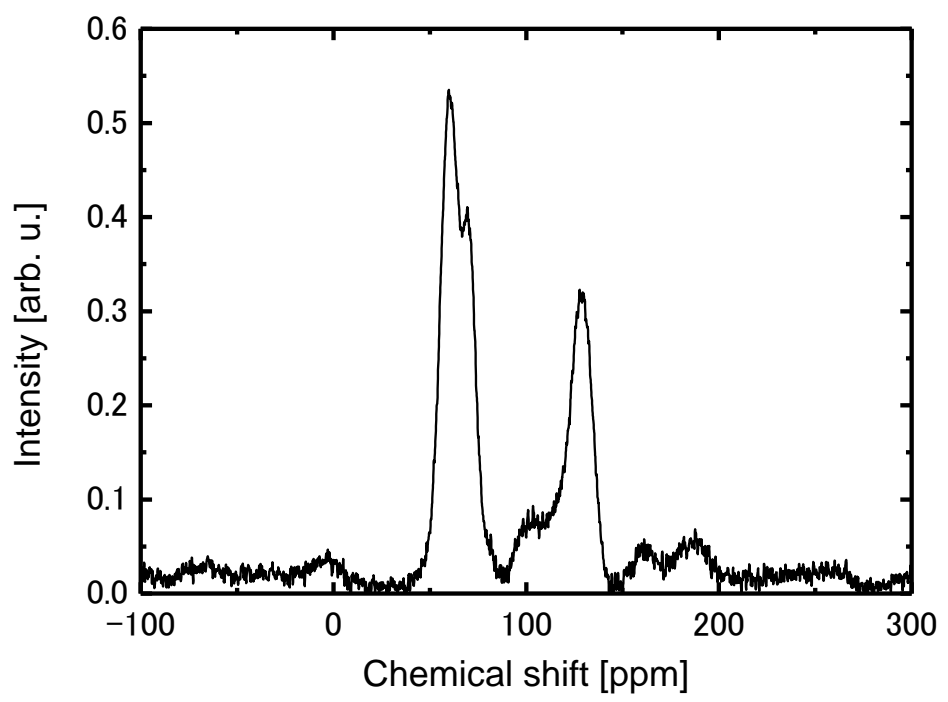

Figure S9. Solid-state ${ }^{13} \mathrm{C}$ NMR spectrum of GO. The peaks at the chemical shifts of 60 , 70 , and $130 \mathrm{ppm}$ correspond to $\mathrm{C}-\mathrm{O}-\mathrm{C}, \mathrm{C}-\mathrm{OH}$, and the $\mathrm{sp}^{2}$ carbon, respectively. ${ }^{\mathrm{S}}$ 

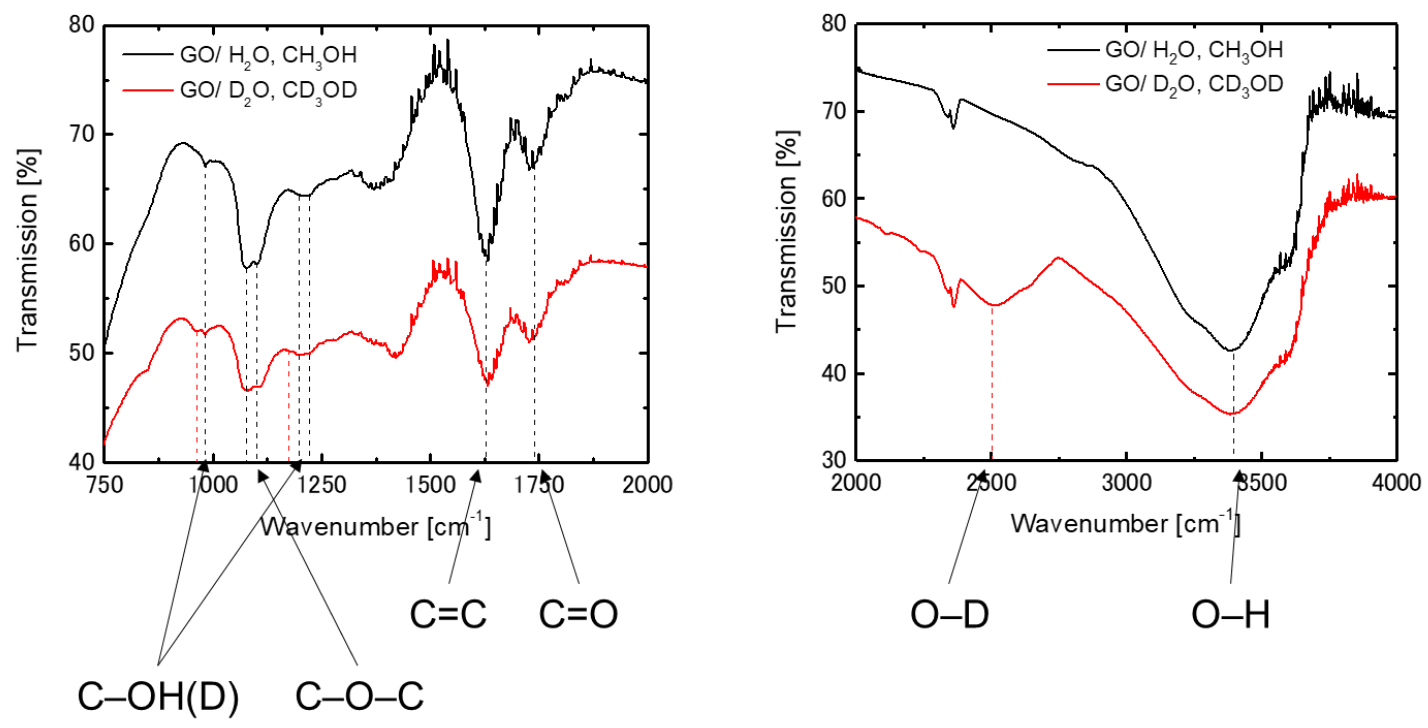

Figure S10. Fourier transform infrared spectrum of GO. Each peak is assigned to a corresponding bond, as indicated. ${ }^{\mathrm{S} 6}$ The vibrational modes in the fingerprint region (wavenumbers of $800-1300 \mathrm{~cm}^{-1}$ ) are related to $\mathrm{C}-\mathrm{C}$ or $\mathrm{C}-\mathrm{O}$ bonds. We compared the spectra of $\mathrm{GO}$ dispersed in $\mathrm{H}_{2} \mathrm{O} / \mathrm{CH}_{3} \mathrm{OH}$ solution and $\mathrm{D}_{2} \mathrm{O} / \mathrm{CD}_{3} \mathrm{OD}$ solution to classify the peaks in the finger-print region. During the drying process, some of the $\mathrm{D}$ atoms in $\mathrm{GO}$ dispersed in the $\mathrm{D}_{2} \mathrm{O} / \mathrm{CD}_{3} \mathrm{OD}$ solution were also replaced by $\mathrm{H}$ atoms. The vibrational peak assignment is summarized in Table $\mathrm{S} 1$. 
Table S1. Vibrational peak assignment in IR spectra

\begin{tabular}{|c|c|c|}
\hline No. & $k_{\text {obs }}\left[\mathrm{cm}^{-1}\right]$ & Functional group \\
\hline 1 & 982 & GO-OH, hydroxyl group on GO \\
2 & 1075 & GO-O, epoxy group on GO \\
3 & 1100 & GO-O, epoxy group on GO \\
4 & 1200 & GO-OH, hydroxyl group on GO \\
5 & 1220 & GO-OH, hydroxyl group on GO \\
6 & 1630 & $\mathrm{C}=\mathrm{C}$ in GO \\
7 & 1735 & $\mathrm{C}=\mathrm{O}$, carbonyl group on GO \\
8 & 3400 & O-H in GO \\
\hline
\end{tabular}




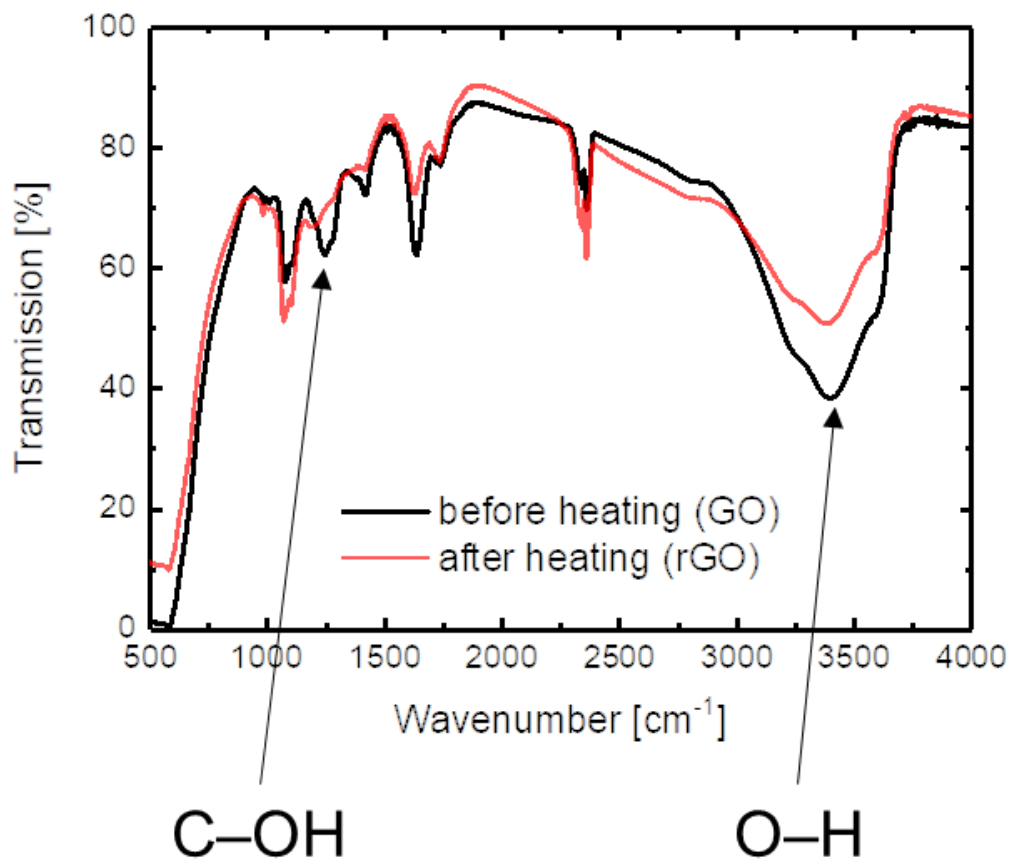

Figure S11. Fourier transform infrared spectra of GO before and after heating (423 K). After heating, the vibrational peak related to the $\mathrm{O}-\mathrm{H}$ bonds $\left(3400 \mathrm{~cm}^{-1}\right)$ decreases, and the peaks related to the $\mathrm{GO}-\mathrm{OH}$ bonds $\left(1200\right.$ and $\left.1220 \mathrm{~cm}^{-1}\right)$ decrease and shift. 


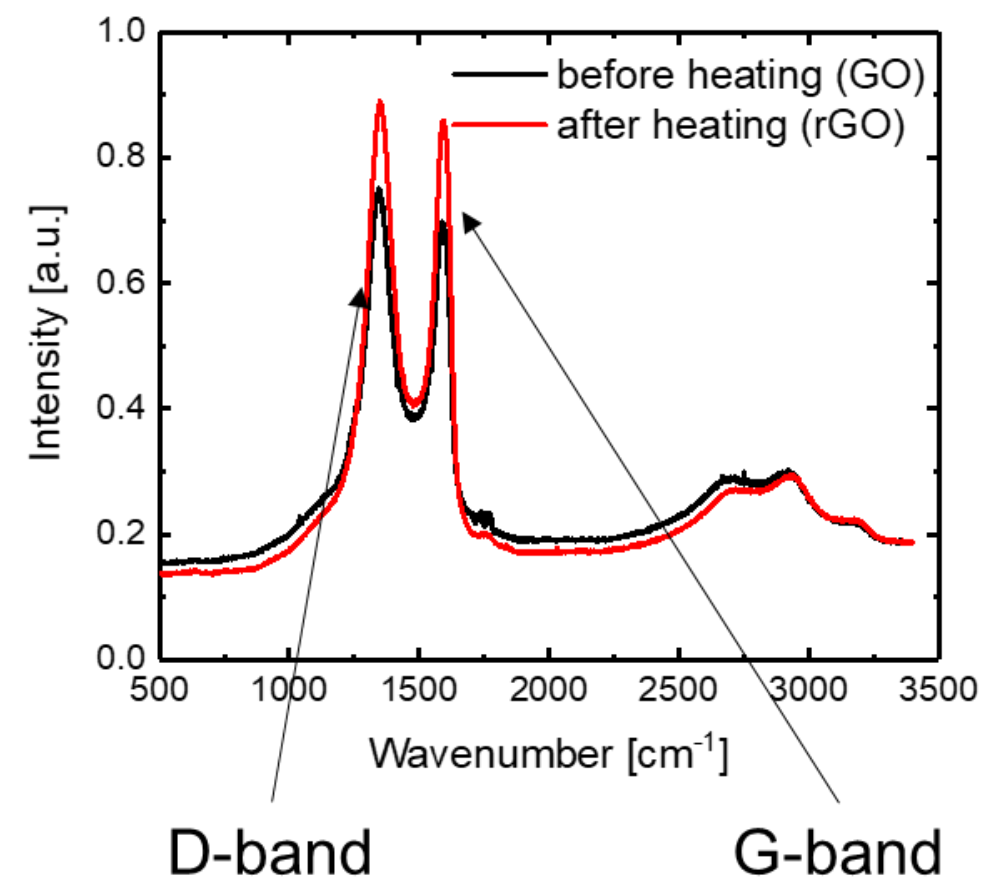

Figure S12. Raman spectra of GO before and after heating (423 K). The peaks corresponding to the D-band $\left(1360 \mathrm{~cm}^{-1}\right)$ and G-band $\left(1590 \mathrm{~cm}^{-1}\right)$ in GO are observed before and after heating. 


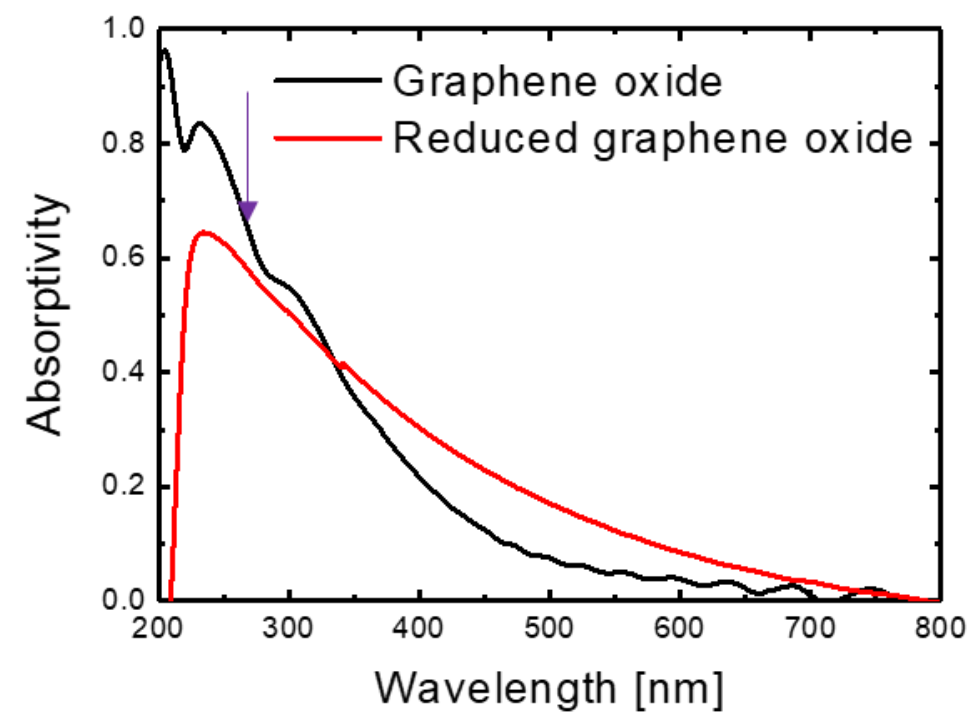

Figure S13. UV-visible spectrum (V-670, Jasco) of GO and thermally reduced GO featuring a strong $\pi \rightarrow \pi^{*}$ band in the UV range. The thickness $(d)$ of the films was 350 $\mathrm{nm}$. The employed photoexcitation wavelength $(266 \mathrm{~nm})$ is indicated by the purple arrow $(\sim 65 \%)$. According to the following equation, the linear absorption coefficient $(l)$ at a wavelength of $266 \mathrm{~nm}$ is $330 \mathrm{~nm}$ :

$$
T=1-A=\exp \left(-\frac{d}{l}\right)
$$

where $T$ and $A$ are the transmission and absorptivity, respectively. For ultrafast timeresolved electron diffraction, the sample thickness was $70 \mathrm{~nm}$, and the absorptivity of the light at a wavelength of $266 \mathrm{~nm}$ was $19 \%$. For the pump light at a fluence of $2 \mathrm{~mJ} / \mathrm{cm}^{2}$, we increased the temperature of GO by $54 \mathrm{~K}$, and we used a density of $1.1 \mathrm{~g} / \mathrm{cm}^{3}$ and a specific heat of $1 \mathrm{~J} / \mathrm{g} \cdot \mathrm{K}$. For the time-resolved IR vibrational spectroscopy, the sample thickness was $0.7 \mu \mathrm{m}$ and the absorptivity of the light at a wavelength of $266 \mathrm{~nm}$ was $88 \%$. 


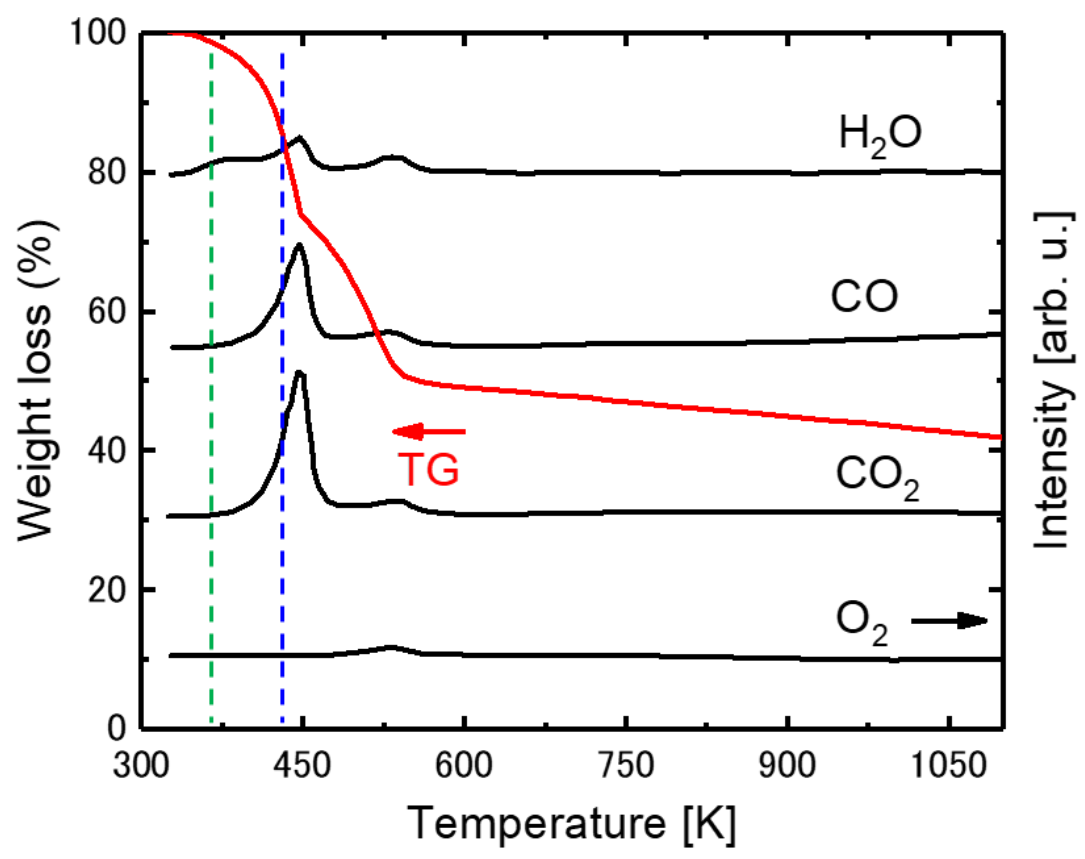

Figure S14. Results of thermogravimetric (TG) and mass-spectrometry (MS) analyses of GO at increasing temperatures. The loss of adsorbed water molecules at $\sim 370 \mathrm{~K}$ is indicated by a green dashed line, whereas the loss of water, carbon monoxide, and carbon dioxide at $\sim 420 \mathrm{~K}$ is indicated by a blue dashed line. TG-MS spectra were recorded with a RIGAKU Thermo plus EVO2 analyzer coupled to a Shimadzu GC-MS QP2010 instrument using $\mathrm{He}(99.999 \%)$ as a carrier gas $(300 \mathrm{~mL} / \mathrm{min})$ and a heating rate of $6^{\circ} \mathrm{C} / \mathrm{min}$. The MS measurement was performed in the SIM mode to detect target molecules. 


\section{DFT and MM2 calculations for graphene oxide}

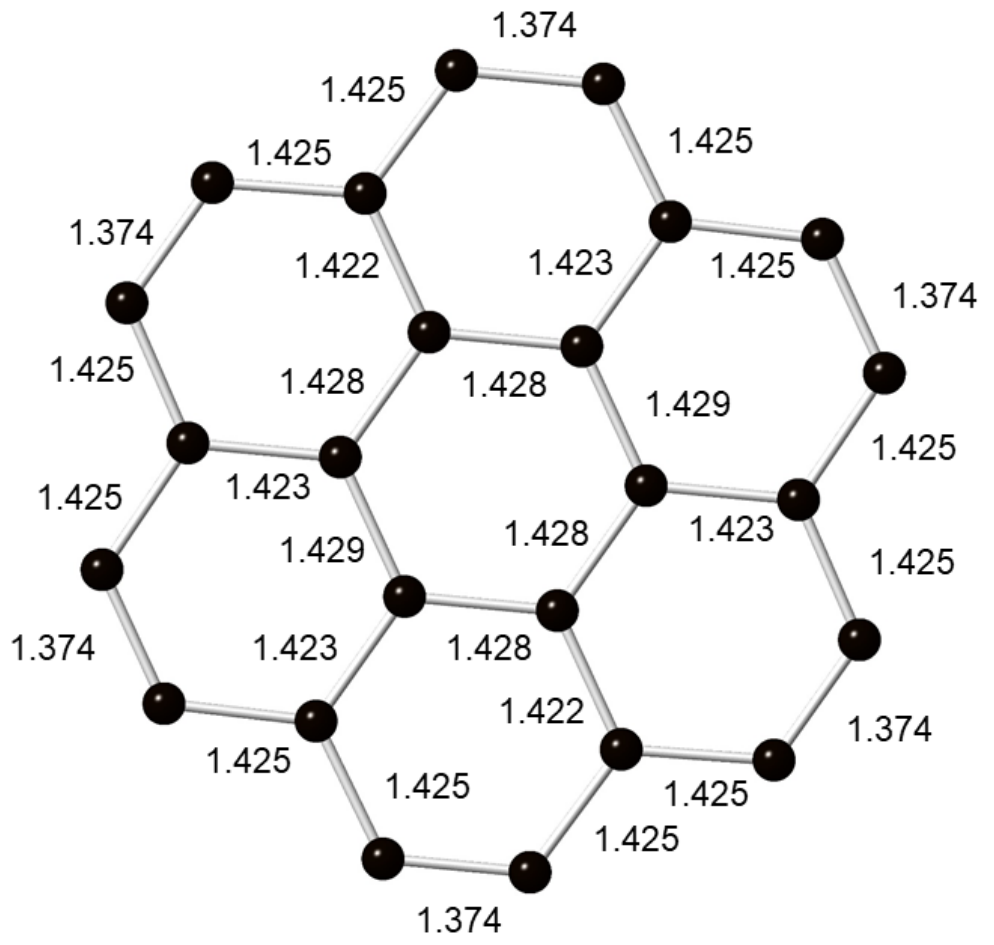

Figure S15. Model of the graphene structure base on DFT calculations featuring bond lengths (in $\AA$ ) obtained from B3LYP/6-31G** level calculations. The average bond length was determined to be $1.415 \AA$, and black spheres indicate carbon atoms. 

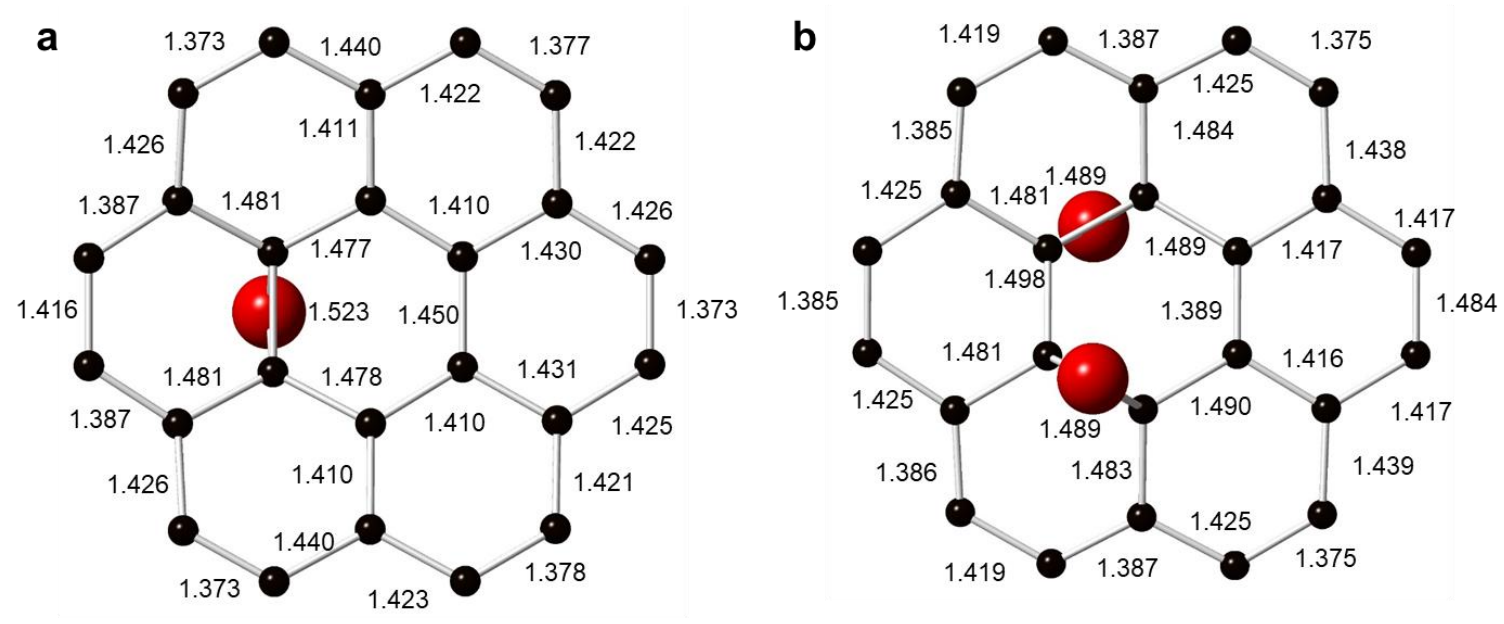

Figure S16. Models of GO structures obtained using DFT calculations featuring one (a) or two (b) oxygen atoms present in epoxy functional groups, and the bond lengths (in $\AA$ ) obtained from B3LYP/6-31G** level calculations are shown. The average bond lengths of S2a and S2b were determined to be 1.424 and $1.434 \AA$, respectively. Red spheres indicate oxygen atoms. 

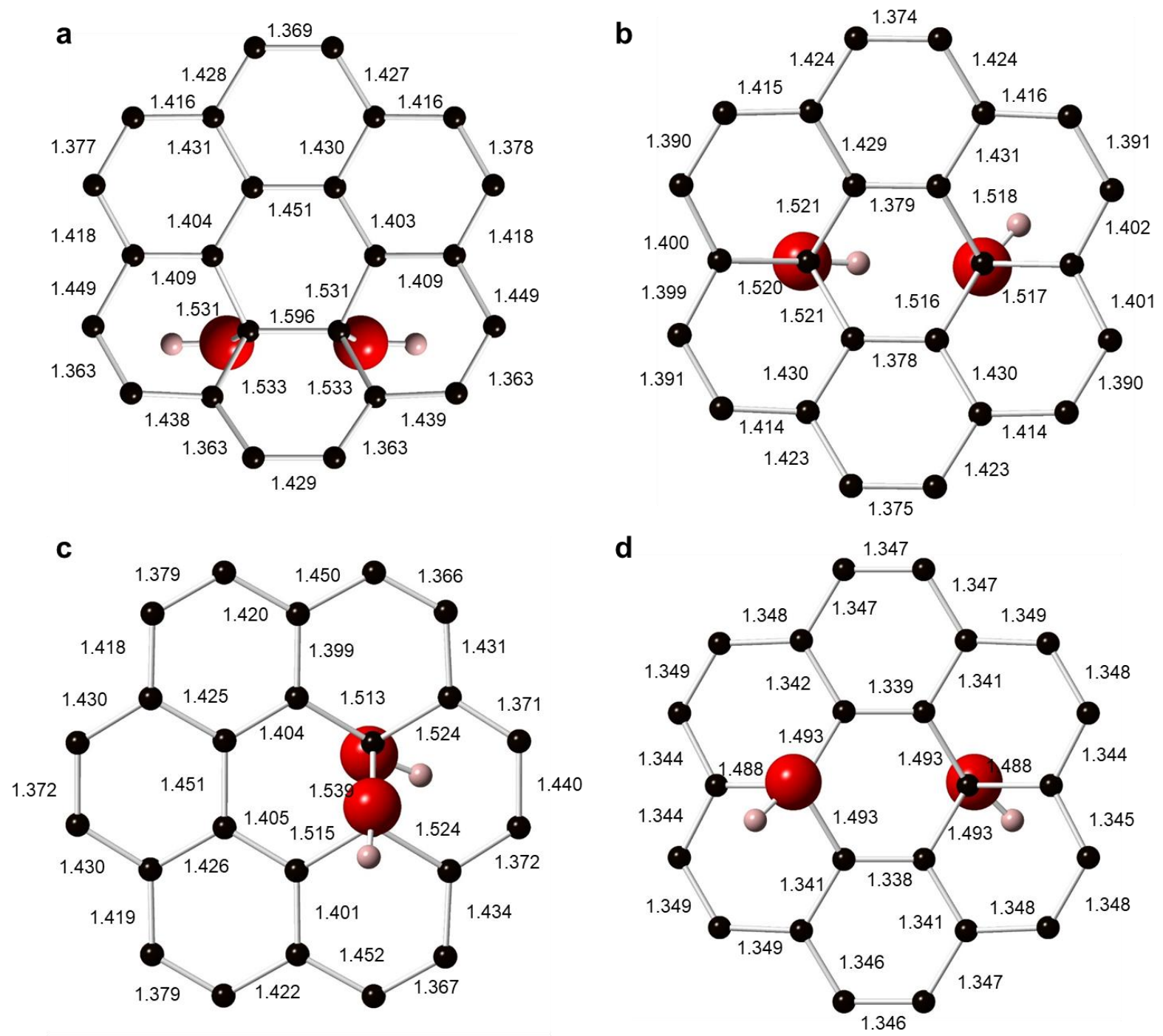

Figure S17. Models of GO structures obtained using DFT calculations featuring oxygen atoms present in hydroxyl groups, and the bond lengths (in $\AA$ ) obtained from B3LYP/6$31 \mathrm{G}^{* *}$ level calculations are shown. The average bond length was determined to be 1.417 Å. Pink spheres indicate hydrogen atoms. 


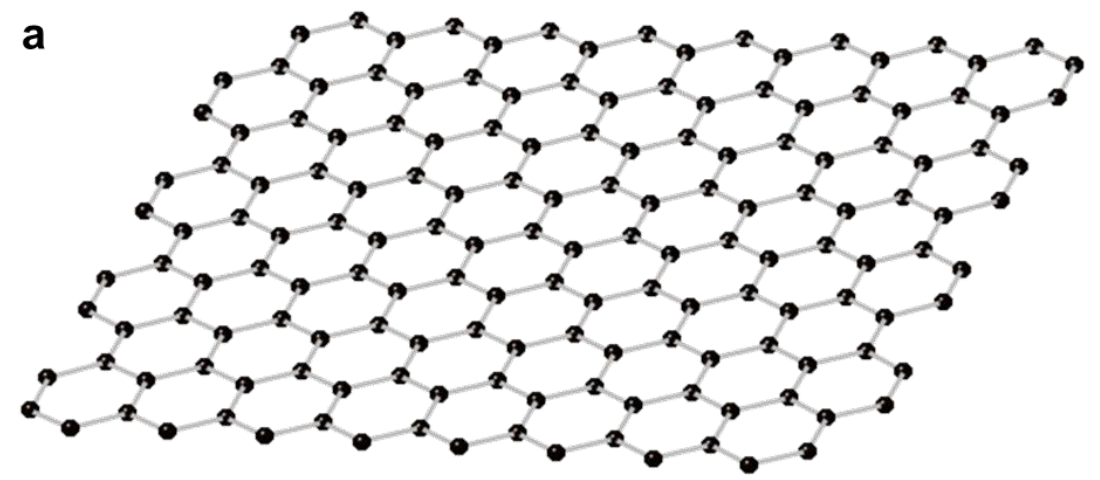

b

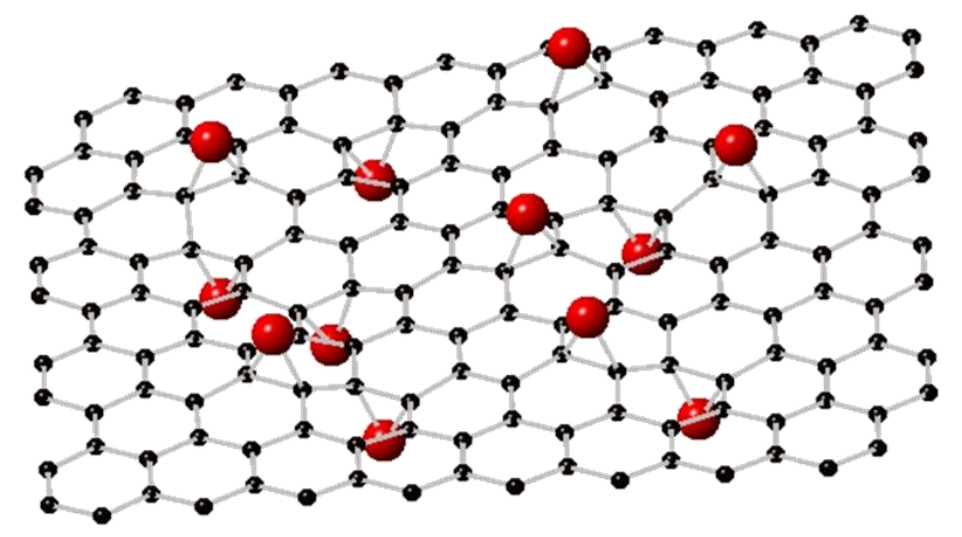

c

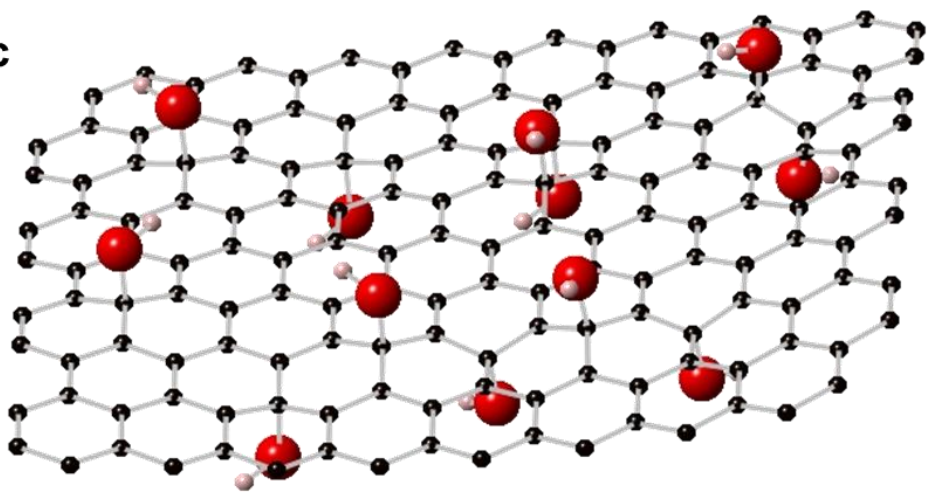

Figure S18. Models of the structure of (a) graphene, (b) GO with oxygen atoms present in epoxy groups, and (c) GO with oxygen atoms present in hydroxyl groups used for MM2 calculations. 


\section{Transient absorption of graphene oxide}
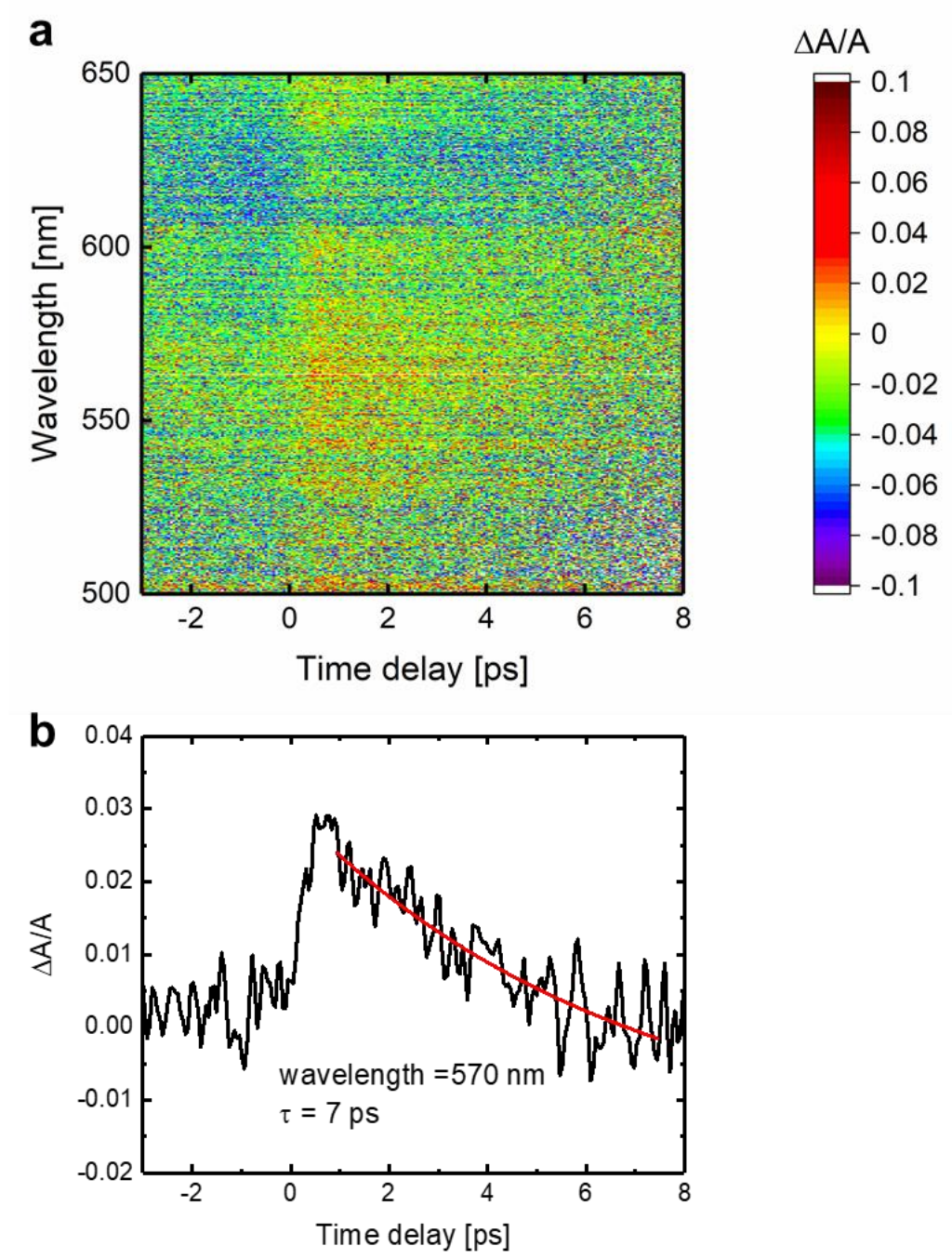

Figure S19. Single shot transient absorption spectra. (a) Two-dimensional map of the single shot transient absorption as functions of the time delay and wavelength. The details of the experiment are provided elsewhere. ${ }^{\mathrm{S} 7}$ The incident fluence of the UV pulse (wavelength of $266 \mathrm{~nm}$ and pulse duration of $120 \mathrm{fs}$ ) was set to $1.5 \mathrm{~mJ} / \mathrm{cm}^{2}$. (b) Transient absorption spectrum at a wavelength of $570 \mathrm{~nm}$. The red line is the fit obtained using an exponential decay curve with a decay time of 7 ps. 

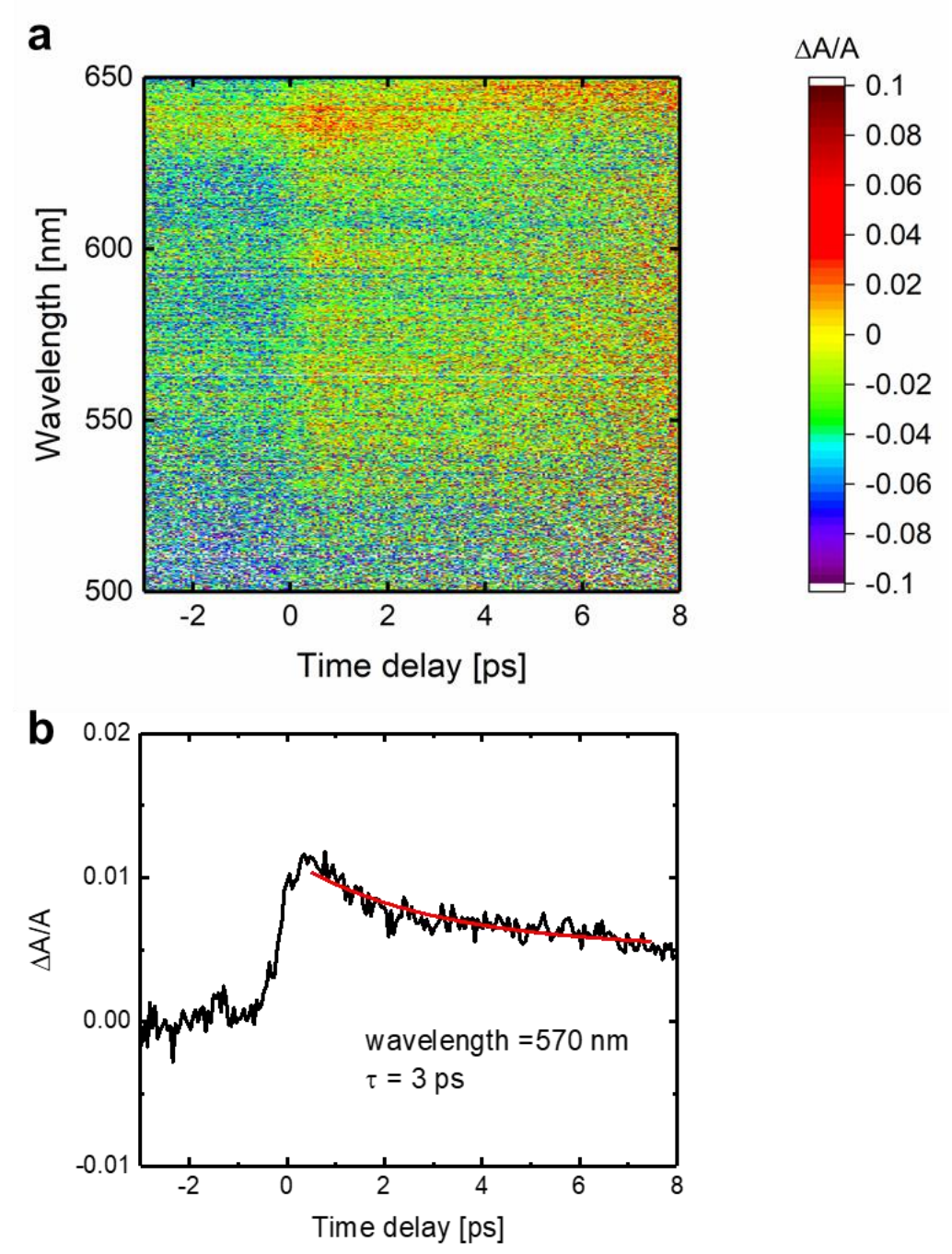

Figure S20. Multiple shot (100 shots) transient absorption spectra. (a) Two-dimensional map of repetitive transient absorption as functions of the time delay and wavelength. One hundred pump-probe experiments were repetitively performed on the same sample spot to acquire an image. The incident fluence of the UV pulse (wavelength of $266 \mathrm{~nm}$ and pulse duration of $120 \mathrm{fs}$ ) was set to $0.75 \mathrm{~mJ} / \mathrm{cm}^{2}$. (b) Transient absorption spectrum at a wavelength of $570 \mathrm{~nm}$. The red line is the fit obtained using an exponential decay curve with a decay time of 3 ps and a constant offset, probably due to components with long decay times. 


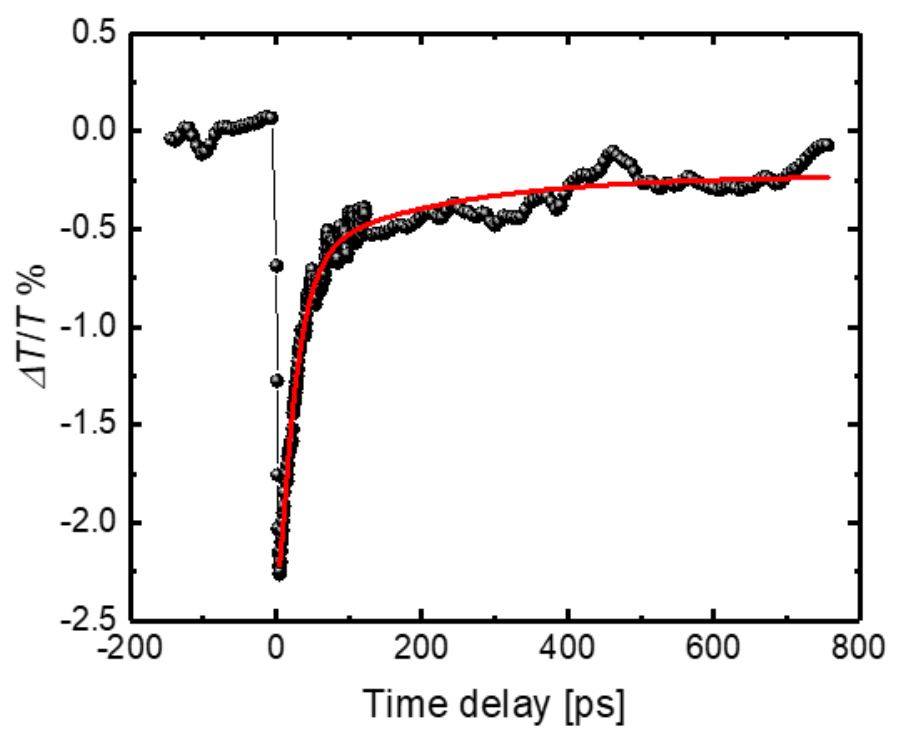

Figure S21. Transient transmission spectrum obtained from repeated measurements. Transient transmission experiments with pump and probe pulse wavelengths of $266 \mathrm{~nm}$ (third harmonic generation of Ti: sapphire regenerative laser) and $400 \mathrm{~nm}$ (second harmonic generation of Ti: sapphire regenerative laser), respectively, were performed. The incident fluence, pulse duration, and repetition of the pump UV pulses were $2 \mathrm{~mJ} / \mathrm{cm}^{2}$, $\sim 100 \mathrm{fs}$, and $500 \mathrm{~Hz}$, respectively. The pulse duration and repetition rate of the probe pulses were $\sim 100 \mathrm{fs}$ and $1 \mathrm{kHz}$, respectively. The signal of the probe light moderated at $500 \mathrm{~Hz}$ was detected using a GaP photodetector and a lock-in amplifier. The red line is the fit obtained using a double exponential decay curve with a long decay component of 205 ps. 


\section{TD-DFT calculations}

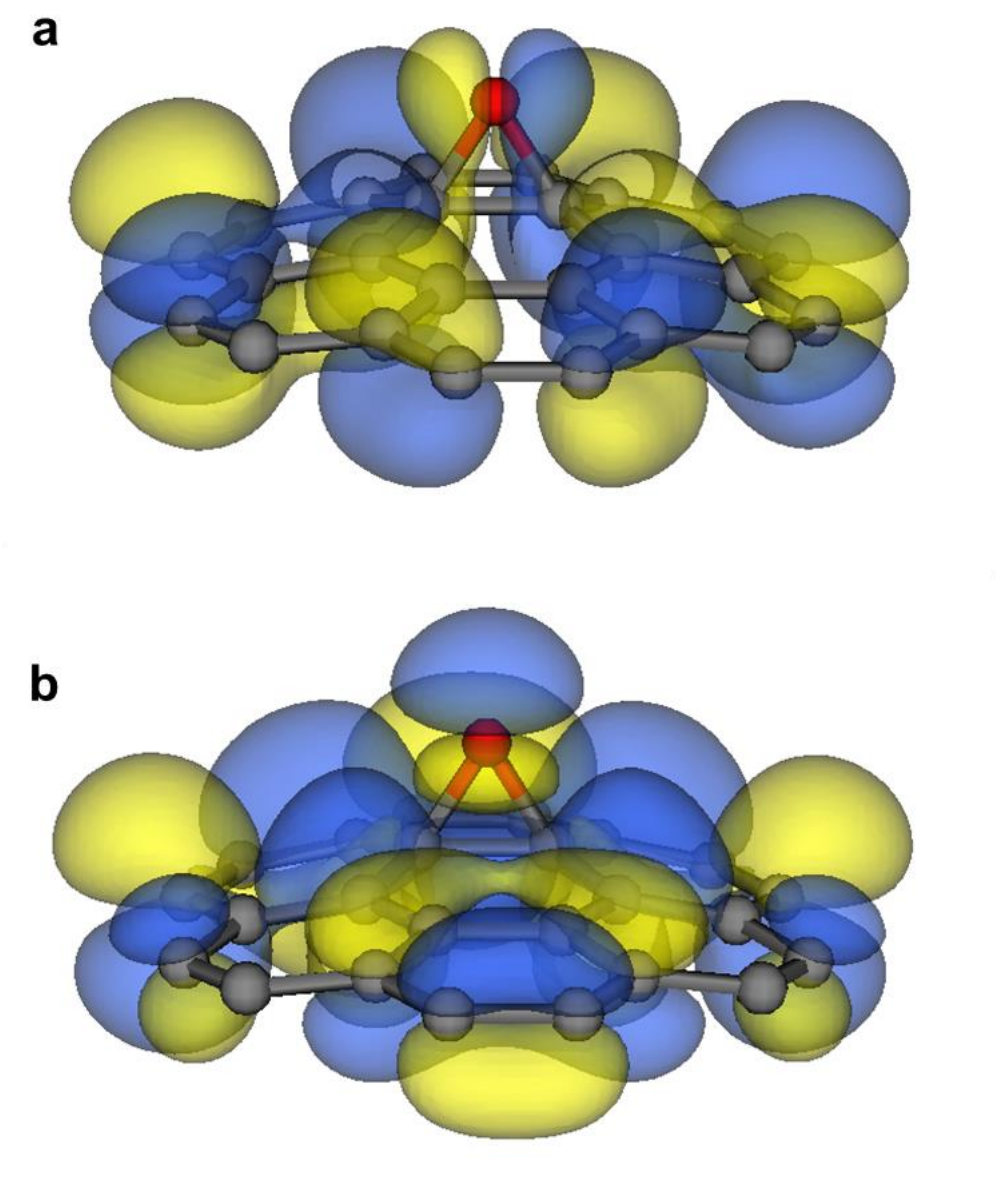

Figure S22. The spatial distributions of the wave functions of GO models (24 carbon atoms) with an epoxy oxygen in the HOMO (a) and LUMO (b). The yellow and blue colors represent isosurfaces with values of 0.015 a.u. and -0.015 a.u., respectively. The calculated $\mathrm{C}-\mathrm{O}$ bond lengths of epoxy oxygen are presented in the main text (Figure 7e). 

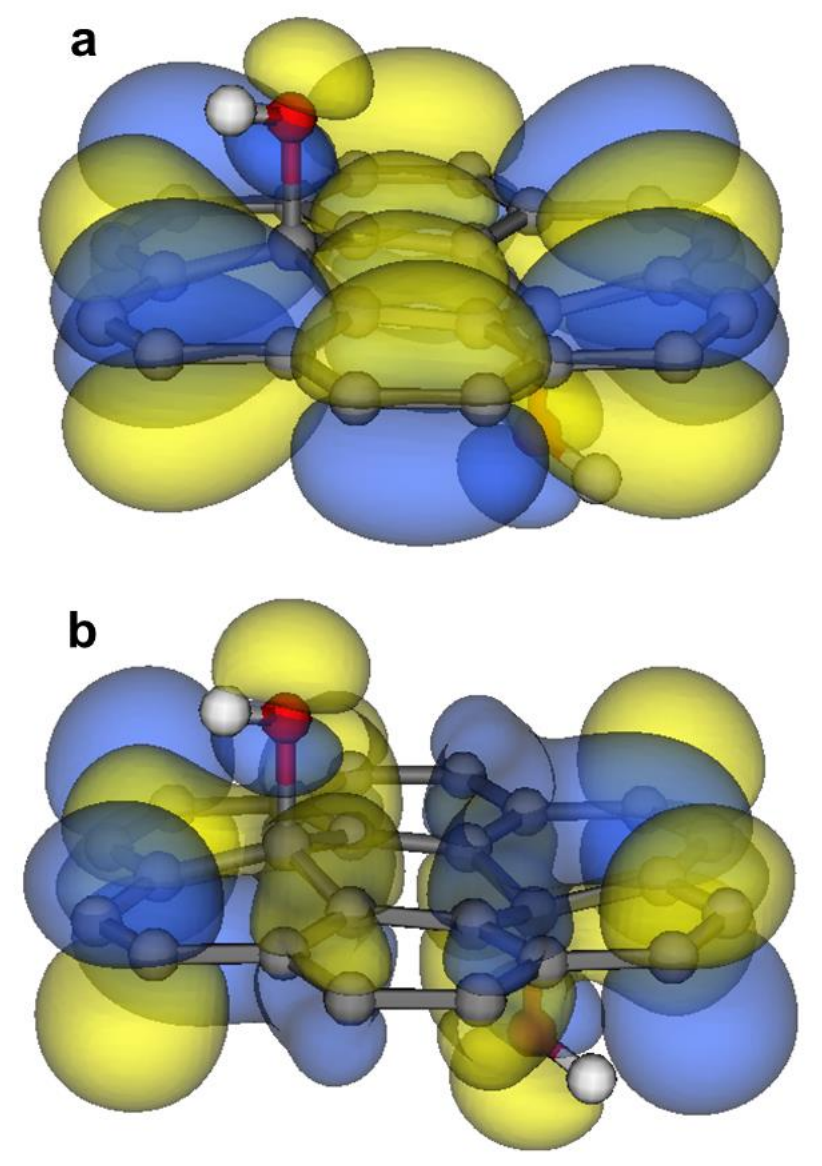

Figure S23. The spatial distributions of the wave functions of GO models (24 carbon atoms) with hydroxyl oxygens in the HOMO (a) and LUMO (b). The yellow and blue colors represent isosurfaces with values of 0.015 a.u. and -0.015 a.u., respectively. The calculated $\mathrm{C}-\mathrm{O}$ bond lengths of hydroxyl oxygen are presented in the main text (Figure 7f). 

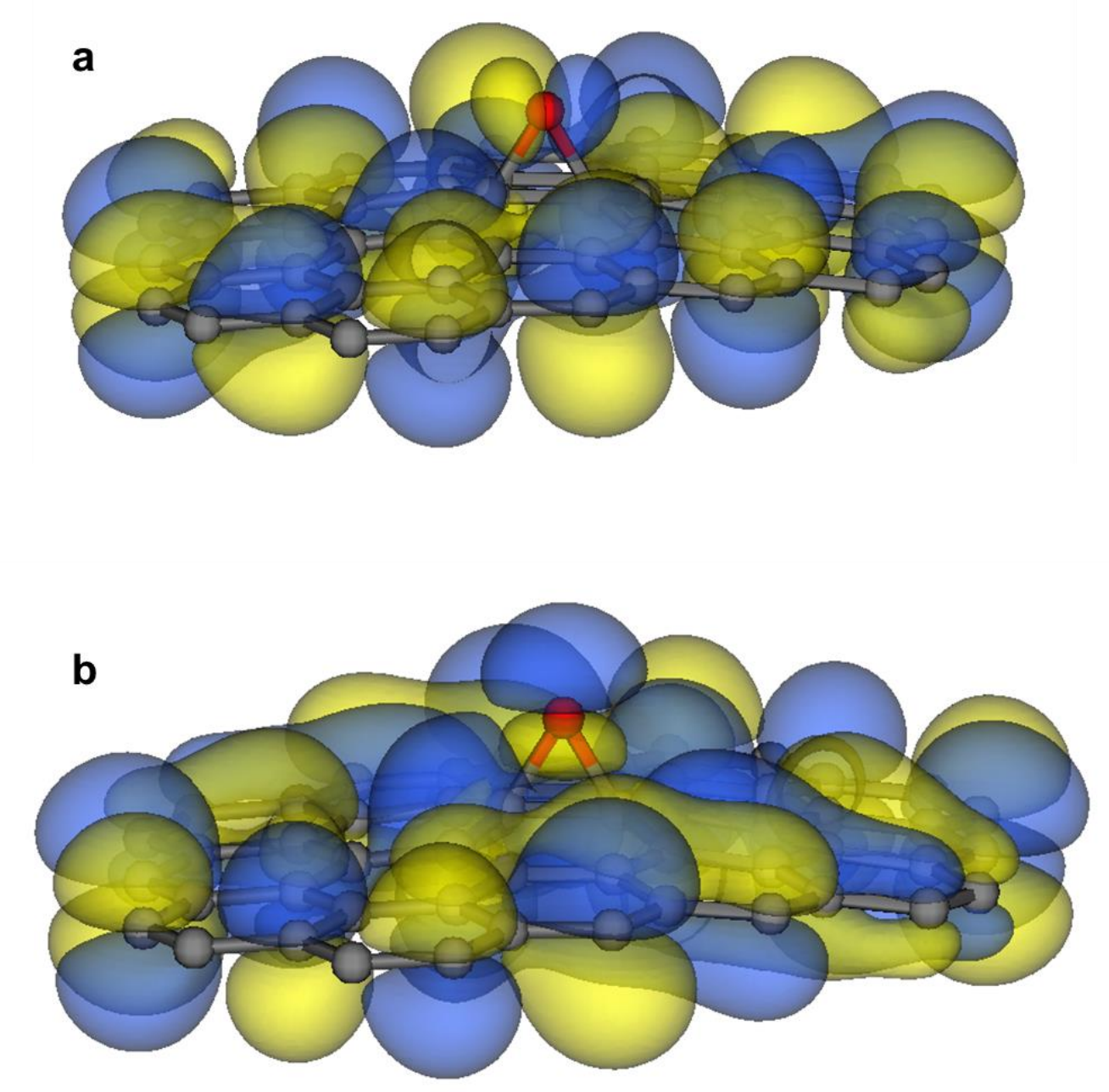

Figure S24. The spatial distributions of the wave functions of GO models (52 carbon atoms) with an epoxy oxygen in the HOMO (a) and LUMO (b). The yellow and blue colors represent isosurfaces with values of 0.015 a.u. and -0.015 a.u., respectively. The spatial distribution of the wave function essentially does not change with the size of the GO model. 

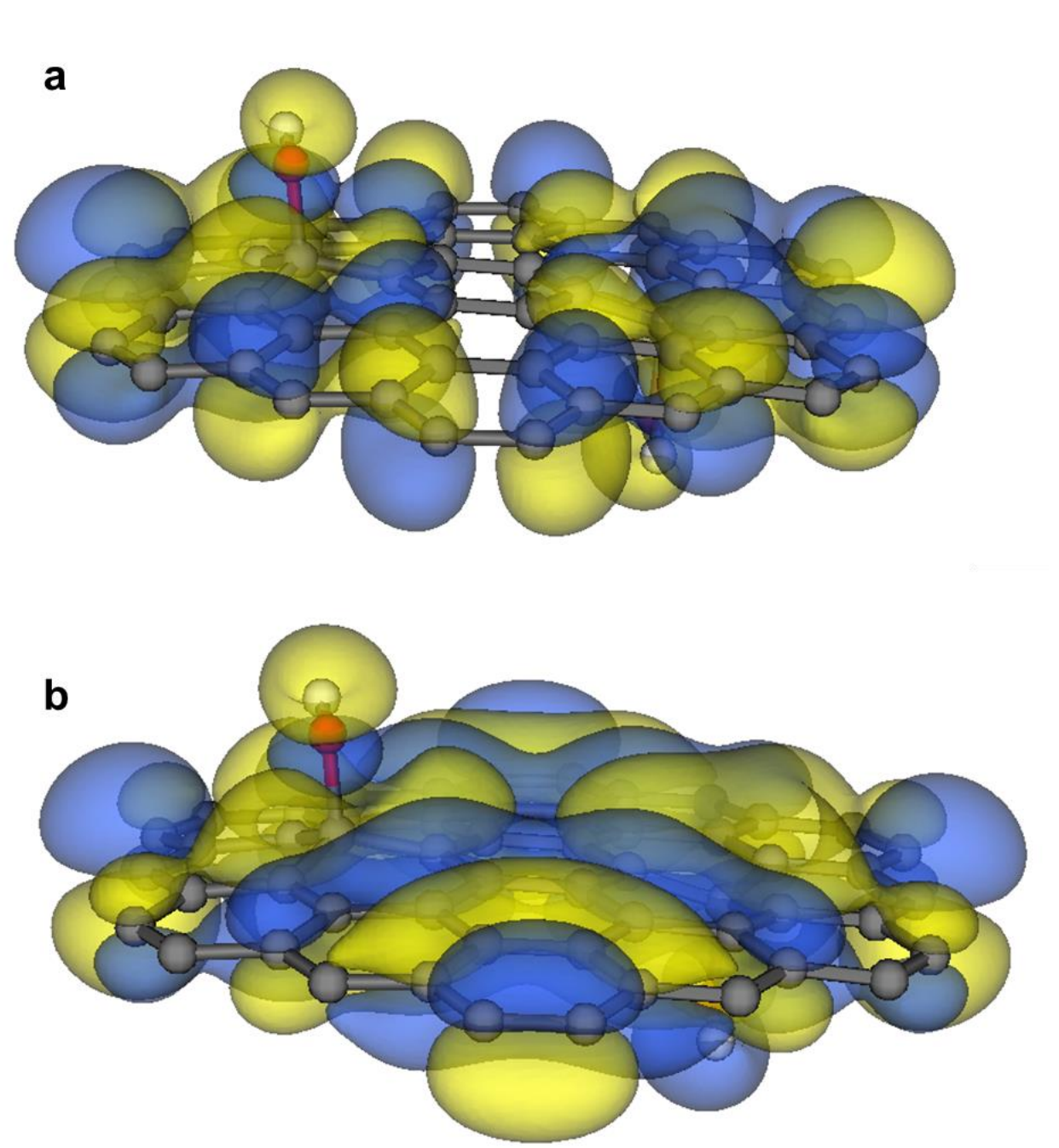

Figure S25. The spatial distributions of the wave functions of GO models (54 carbon atoms) with hydroxyl oxygens in the HOMO (a) and LUMO (b). The yellow and blue colors represent isosurfaces with values of 0.015 a.u. and -0.015 a.u., respectively. The spatial distribution of the wave function essentially does not change with the size of the GO model. 


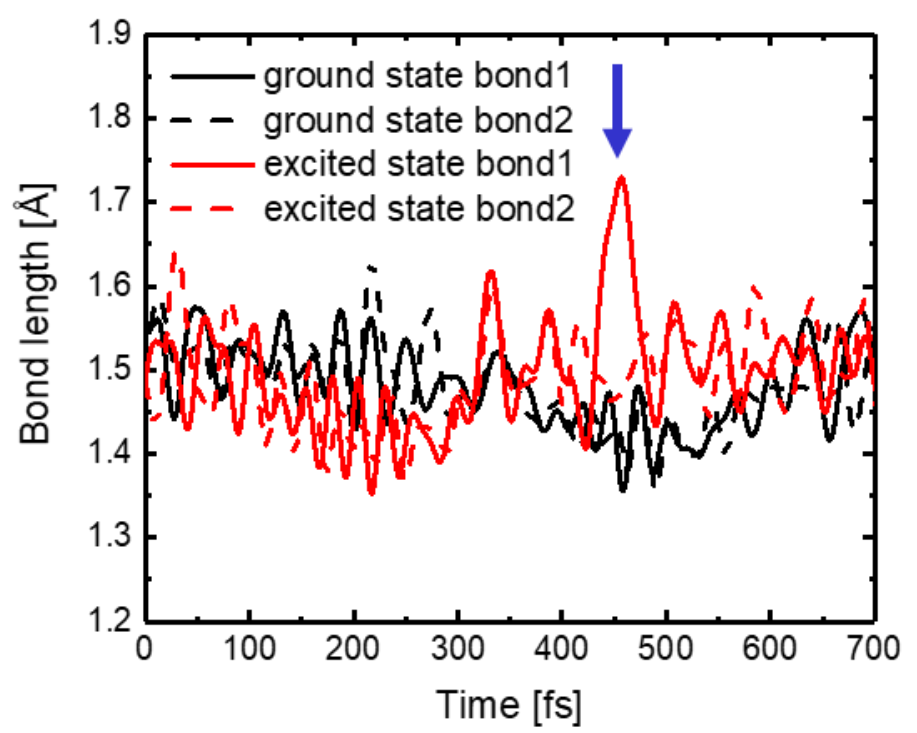

Figure S26. Calculated $\mathrm{C}-\mathrm{O}$ bond lengths for an epoxy oxygen atom in the GO model shown in Figure S21 with and without excitation. The $\mathrm{C}-\mathrm{O}$ bond is weakened in the excited state due to the anti-bonding character, as indicated by the blue arrow. 


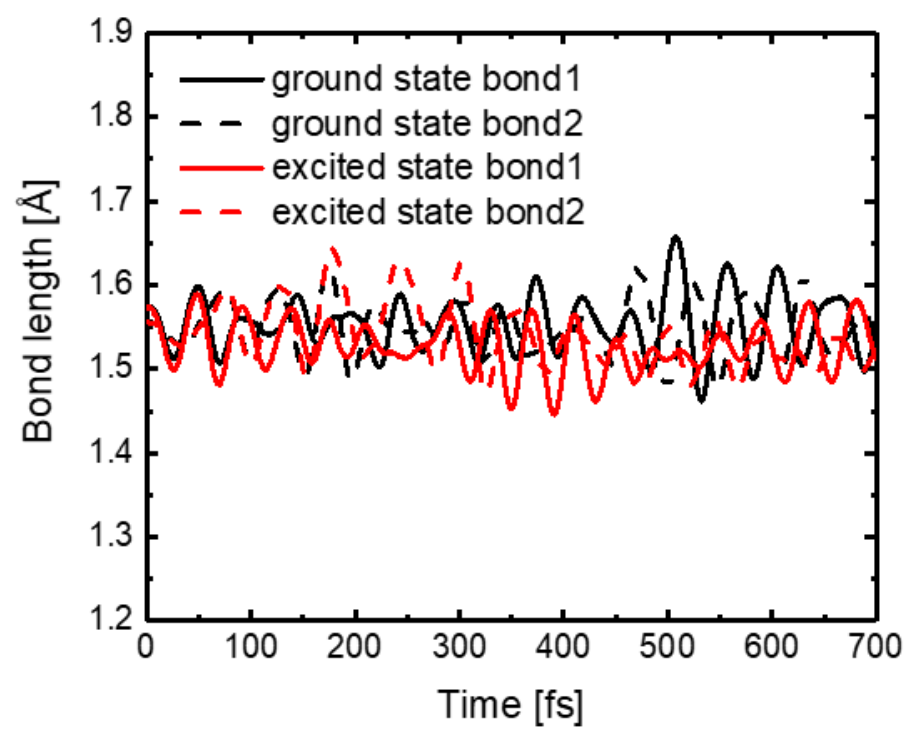

Figure $\mathrm{S} 27$. Calculated $\mathrm{C}-\mathrm{O}$ bond lengths for hydroxyl oxygen atoms in the $\mathrm{GO}$ model shown in Figure $\mathrm{S} 22$ with and without excitation. The $\mathrm{C}-\mathrm{O}$ bond is not weakened in the excited state. 


\section{Note S1}

\section{Numerical details}

The electronic states were calculated using the projected-augmented wave $(\mathrm{PAW})^{\mathrm{S} 8, \mathrm{~S} 9}$ method within the framework of density functional theory (DFT). We included the $2 \mathrm{~s}$ and $2 \mathrm{p}$ states of $\mathrm{C}$ and $\mathrm{O}$ and the $1 \mathrm{~s}$ state of $\mathrm{H}$ as the valence electrons. The generalized gradient approximation (GGA-PBE) ${ }^{\mathrm{S} 10}$ was used to calculated the exchange-correlation energy. The electronic pseudowave functions and the pseudocharge density are expanded by plane waves with cutoff energies of 30 and $250 \mathrm{Ry}$, respectively. The energy functional is minimized with respect to the Kohn-Sham (KS) orbitals using an iterative method. ${ }^{\mathrm{S} 11, \mathrm{~S} 12}$

We describe excited electronic states as linear combinations of electron-hole pairs within Casida's linear-response time-dependent density functional theory (LRTDDFT), ${ }^{\text {S13-S16 }}$ using the ground-state KS orbitals as a basis set. In LR-TDDFT, electronic excitation energies are calculated from the poles of an electron-hole pair response function. This procedure amounts to solving an eigenvalue problem, with a matrix size of $N_{\mathrm{o}} N_{\mathrm{u}} \times N_{\mathrm{o}} N_{\mathrm{u}}$ when using the GGA ( $N_{\mathrm{o}}$ and $N_{\mathrm{u}}$ are the numbers of occupied and unoccupied KS orbitals, respectively, used to represent excited states). In this study, we included HOMO-4 HOMO as occupied states and LUMO LUMO+4 as unoccupied states. Here, many-body effects are introduced by coupling matrix elements consisting of the random-phase-approximation and exchange-correlation terms.

Molecular dynamics (MD) simulations of the canonical ensemble were performed using the Nosè-Hoover thermostat technique. ${ }^{\mathrm{S} 17, \mathrm{~S} 18}$ The equations of motion were integrated numerically using an explicit reversible integrator ${ }^{\mathrm{S} 19}$ with a time step of 20 a.u. ( $0.48 \mathrm{fs})$. All MD simulations were performed at room temperature. 


\section{Supporting References}

S1. Nozawa, S.; Adachi, S.; Takahashi, J.; Tazaki, R.; Guérin, L.; Daimon, M.; Tomita, A.; Sato, T.; Chollet, M.; Collet, E.; Cailleau, H.; Yamamoto, S.; Tsuchiya, K.; Shioya, T.; Sasaki, H.; Mori, T.; Ichiyanagi, K.; Sawa, H.; Kawata, H.; Koshihara, S. Developing 100 Ps-Resolved X-ray Structural Analysis Capabilities on Beamline NW14A at the Photon Factory Advanced Ring. J. Synchrotron Radiat. 2007, 14, 313-319.

S2. Ichiyanagi, K.; Sato, T.; Nozawa, S.; Kim, K. H.; Lee, J. H.; Choi, J.; Tomita, A.; Ichikawa, H.; Adachi, S.; Ihee, H.; Koshihara, S. 100 Ps Time-Resolved Solution Scattering Utilizing a Wide-Bandwidth X-ray Beam from Multilayer Optics. $J$. Synchrotron Radiat. 2009, 16, 391-394.

S3. Gómez-Navarro, C.; Meyer, J. C.; Sundaram, R. S.; Chuvilin, A.; Kurasch, S.; Burghard, M.; Kern, K.; Kaiser, U. Atomic Structure of Reduced Graphene Oxide. Nano Lett. 2010, 10, 1144-1148.

S4. Wyckof, R. W. G. In Crystal Structures, 2nd edition; Interscience publishers: New York, 1963; pp. 7-83.

S5. Morioku, K.; Morimoto, N.; Takeuchi, Y.; Nishina, Y. Concurrent Formation of Carbon-Carbon Bonds and Functionalized Graphene by Oxidative CarbonHydrogen Coupling Reaction. Sci. Rep. 6, 25824 (2016).

S6. Su, C.; Loh, K. P. Carbocatalysts: Graphene Oxide and Its Derivatives. Acc. Chem. Res. 2013, 46, 2275-2285.

S7. Takeda, J.; Oba, W.; Minami, Y.; Saiki, T.; Katayama I. Ultrafast Crystalline-toAmorphous Phase Transition in $\mathrm{Ge}_{2} \mathrm{Sb}_{2} \mathrm{Te}_{5}$ Chalcogenide Alloy Thin Film Using Single-Shot Imaging Spectroscopy. Appl. Phys. Lett. 2014, 104, 261903.

S8. Blöchl, P. E. Projector Augmented-Wave Method. Phys. Rev. B 1994, 50, 1795317979.

S9. Kresse, G.; Joubert, D. From Ultrasoft Pseudopotentials to the Projector Augmented-Wave Method. Phys. Rev. B 1999, 59, 1758-1775.

S10. Perdew, J. P.; Burke, K.; Ernzerhof, M. Generalized Gradient Approximation Made Simple. Phys. Rev. Lett. 1996, 77, 3865-3868.

S11. Kresse, G.; Hafner, J. Ab Initio Molecular-Dynamics Simulation of the LiquidMetal-Amorphous-Semiconductor Transition in Germanium. Phys. Rev. B 1994, 49, 14251-14269.

S12. Shimojo, F.; Kalia, R. K.; Nakano, A.; Vashishta, P. Linear-Scaling DensityFunctional-Theory Calculations of Electronic Structure Based on Real-Space 
Grids: Design, Analysis, and Scalability Test of Parallel Algorithms. Comput. Phys. Commun. 2001, 140, 303-314.

S13. Casida, M. E. Recent Advances in Computational Chemistry: Volume 1. In Recent Advances in Density Functional Methods (part I); Chong, D. P., Ed.; World Scientific: Singapore, 1995; pp. 155-192.

S14. Hirai, H.; Sugino, O. A Time-Dependent Density-Functional Approach to Nonadiabatic Electron-Nucleus Dynamics: Formulation and Photochemical Application. Phys. Chem. Chem. Phys. 2009, 11, 4570-4578.

S15. Walter, M.; Häkkinen, H.; Lehtovaara, L.; Puska, M.; Enkovaara, J.; Rostgaard, C.; Mortensen, J. J. Time-Dependent Density-Functional Theory in the Projector Augmented-Wave Method. J. Chem. Phys. 2008, 128, 244101.

S16. Tapavicza, E.; Tavernelli, I.; Rothlisberger, U. Trajectory Surface Hopping within Linear Response Time-Dependent Density-Functional Theory. Phys. Rev. Lett. 2007, 98, 023001.

S17. Nosé, S. A Molecular Dynamics Method for Simulations in the Canonical Ensemble. Mol. Phys. 1984, 52, 255-268.

S18. Hoover, W. G. Canonical Dynamics: Equilibrium Phase-Space Distributions. Phys. Rev. A 1985, 31, 1695-1697.

S19. Tuckerman, M.; Berne, B. J.; Martyna, G. J. Reversible Multiple Time Scale Molecular Dynamics. J. Chem. Phys. 1992, 97, 1990-2001. 